\title{
NONLINEAR MONOTONE SEMIGROUPS AND VISCOSITY SOLUTIONS
}

\author{
Samuel BITON ${ }^{1}$ \\ Laboratoire de Mathématiques et Physique Théorique, Faculté des Sciences et Techniques, \\ Université de Tours, Parc de Grandmont, 37200 Tours, France
}

Received 30 March 2000

ABSTRACT. - In a celebrated paper motivated by applications to image analysis, L. Alvarez, F. Guichard, P.-L. Lions and J.-M. Morel showed that any monotone semigroup defined on the space of bounded uniformly continuous functions, which satisfies suitable regularity and locality assumptions is in fact a semigroup associated to a fully nonlinear, possibly degenerate, secondorder parabolic partial differential equation. In this paper, we extend this result by weakening the assumptions required on the semigroup to obtain such a result and also by treating the case where the semigroup is defined on a general space of continuous functions like, for example, a space of continuous functions with a prescribed growth at infinity. These extensions rely on a completely different proof using in a more central way the monotonicity of the semigroup and viscosity solutions methods. Then we study the consequences on the partial differential equation of various additional assumptions on the semigroup. Finally we briefly present the adaptation of our proof to the case of two-parameters families.

(C) 2001 L'Association Publications de l'Institut Henri Poincaré. Published by Elsevier B.V. All rights reserved

RÉSUMÉ. - Dans un célèbre article motivé par les applications au traitement d'image, L. Alvarez, F. Guichard, P.-L. Lions and J.-M. Morel ont montré qu'un semi-groupe monotone défini sur l'espace des fonctions bornées uniformément continues satisfaisant des hypothèses de régularité et de localité est en fait un semi-groupe associé à une équation aux dérivées partielles parabolique non linéaire éventuellement dégénérée. Dans le présent article, nous étendons ce résultat en affaiblissant légèrement les hypothèses nécessaires et en traitant le cas de semigroupes définis sur des espaces généraux de fonctions continues. Ces extension résultent d'une preuve totalement différente utilisant de manière plus centrale la monotonie du semi-groupe et des méthodes de solutions de viscosité. Nous étudions ensuite les conséquences d'hypothèses supplémentaires sur le semi-groupe. Finallement, nous présentons brièvement l'adaptation au cas d'une famille d'opérateurs à deux paramètres.

(C) 2001 L'Association Publications de l'Institut Henri Poincaré. Published by Elsevier B.V. All rights reserved

E-mail address: poiton@univ_tours.fr (S. Biton).

${ }^{1}$ This work was partially supported by the TMR program "Viscosity solutions and their applications". 


\section{Introduction}

In this article, we are interested in nonlinear semigroups $\left(T_{t}\right)_{t \geqslant 0}$ defined on some subspace $X \subset \mathcal{C}\left(\mathbb{R}^{N}\right)$ and satisfying the following monotonicity assumption: for any $f, g \in X$

$$
f \leqslant g \Rightarrow T_{t}[f] \leqslant T_{t}[g] \text { for any } t \geqslant 0,
$$

where $\leqslant$ denotes the partial ordering on $\mathcal{C}\left(\mathbb{R}^{N}\right)$ defined by

$$
f \leqslant g \Leftrightarrow f(x) \leqslant g(x) \text { for all } x \in \mathbb{R}^{N} .
$$

In [1], L. Alvarez, F. Guichard, P.-L. Lions and J.-M. Morel show that if $X=$ $B U C\left(\mathbb{R}^{N}\right)$, the space of bounded uniformly continuous functions, and if $\left(T_{t}\right)_{t \geqslant 0}$ satisfies in addition suitable regularity and locality assumptions, such a semigroup is associated to a second-order parabolic partial differential equation. More precisely, they prove that, for any $u_{0} \in B U C\left(\mathbb{R}^{N}\right)$, the continuous function $u(t, x):=T_{t}\left[u_{0}\right](x)$ is a viscosity solution of an initial value problem of the form

$$
\begin{cases}\frac{\partial u}{\partial t}+F\left(x, u, D u, D^{2} u\right)=0 & \text { in }(0,+\infty) \times \mathbb{R}^{N}, \\ u(0, \cdot)=u_{0} & \text { in } \mathbb{R}^{N},\end{cases}
$$

where $D u$ and $D^{2} u$ denote respectively the gradient and the Hessian matrix of $u$, and $F$ is a continuous function on $\mathbb{R}^{N} \times \mathbb{R} \times \mathbb{R}^{N} \times \mathcal{S}(N), \mathcal{S}(N)$ being the space of the $N \times N$ symmetric matrices. We recall that Eq. (1) is said to be (degenerate) parabolic if the function $F$ satisfies the so-called "ellipticity" condition: for all $x \in \mathbb{R}^{N}, r \in \mathbb{R}, p \in \mathbb{R}^{N}$ and $M, N \in \mathcal{S}(N)$,

$$
M \leqslant N \Rightarrow F(x, r, p, M) \geqslant F(x, r, p, N) .
$$

Their proof is essentially done in three steps. The main step is the first one where, using only the regularity assumption on the semigroup together with a contraction property, they show the existence of a nonlinear infinitesimal generator

$$
A[f]=\lim _{t \rightarrow 0^{+}} \frac{T_{t}[f]-f}{t}
$$

which is well-defined if $f$ is smooth. Then, using the monotonicity and the locality assumption, they prove the existence of a continuous function $F$ such that, for any smooth function $f$,

$$
A[f](x)=-F\left(x, f(x), D f(x), D^{2} f(x)\right) \quad \text { in } \mathbb{R}^{N} .
$$

Finally, using again the monotonicity assumption, a classical argument in viscosity solutions theory yields that $u$ is a viscosity solution of (1).

The first contribution of this article is to provide a completely different and, to our opinion, far simpler proof of this result, using in a more fundamental way the 
monotonicity of the semigroup. From the technical point of view, this will mean also that we are also going to use in a more central way viscosity solutions methods. This new proof allows us to weaken slightly the assumptions made in [1] on $\left(T_{t}\right)_{t \geqslant 0}$, to remove the use of a Banach space structure on $X$ and thus to extend the result to a large class of spaces $X$.

Before going further in this introduction, we recall that viscosity solutions are weak solutions for second-order degenerate elliptic partial differential equations and we refer the reader to M.G. Crandall, I. Ishii and P.-L. Lions [6], W. Fleming and H.M. Soner [7] for a complete presentation of this theory and to M. Bardi and I. Capuzzo-Dolcetta [2], G. Barles [3] for an introduction to this theory in the case of first-order equations.

To be more specific on our results, we prove in Section 3, under localized versions of the assumptions used in [1], that there exists a continuous function $F$ such that, for all $x \in \mathbb{R}^{N}$,

$$
\lim _{h \rightarrow 0^{+}} \frac{T_{t}[f](x)-f(x)}{h}=-F\left(x, f(x), D f(x), D^{2} f(x)\right)
$$

for any smooth function $f$ of $X$ and simultaneously that the function $u(t, x):=T_{t}\left[u_{0}\right](x)$ is a viscosity solution of (1) for every $u_{0} \in X$.

Obviously, we need some assumptions on $X$ but it is worth noticing that they are very weak and that they hold for a large class of spaces $X \subset \mathcal{C}\left(\mathbb{R}^{N}\right)$, like $\mathcal{C}\left(\mathbb{R}^{N}\right)$ itself, $B U C\left(\mathbb{R}^{N}\right)$, the space of bounded continuous functions or of continuous functions with growth conditions at infinity, the space of uniformly continuous functions ... etc.

In Section 4, we study the consequences of various additional assumptions on $\left(T_{t}\right)_{t \geqslant 0}$ and describe in particular the consequence of a finite speed of propagation property for $T_{t}[f]$, i.e.

$$
\begin{aligned}
& f(x) \leqslant g(x) \text { for } x \in B\left(x_{0}, r\right) \Rightarrow \exists L_{x_{0}, f, g}>0 \\
& \text { such that } T_{t}[f]\left(x_{0}\right) \leqslant T_{t}[g]\left(x_{0}\right) \text { for } t \leqslant L_{x_{0}, f, g} r^{s} .
\end{aligned}
$$

The fact that a semigroup satisfying such kind of property is a semigroup associated to an Hamilton-Jacobi equation was first proved by P.-L. Lions in [12]. Nevertheless, we give a simplified proof using the Theorem of Section 3 which works in a slightly more general context.

Then we address the following natural question: do the assumptions used on $\left(T_{t}\right)_{t \geqslant 0}$ to connect it to the initial value problem (1) ensure that $u$ is the unique solution of this problem? In [1], the answer was yes; indeed, becauase of the applications to image analysis, it was natural to assume that the semigroup commutes with translations and additions of constants (which yields an $x$ and $u$-independent $F$ ). In this context, a comparison result holds for the viscosity solutions of (1) in $B U C\left(\mathbb{R}^{N}\right)$ (see for example M.G. Crandall, I. Ishii, P-.L. Lions [6] or Y. Giga, S. Goto, I. Ishii and M.-H. Sato [8]).

Here, on the contrary, the answer is no in general, even for a linear semigroup if the assumption of commutation with translations is removed. In Section 5, we build an example of a semigroup defined on $B U C\left(\mathbb{R}^{N}\right)$ which satisfy the assumptions of Section 2 and which is associated to a transport equation of the form

$$
\frac{\partial u}{\partial t}+b(x) \cdot D u=0 \quad \text { in }(0,+\infty) \times \mathbb{R}^{N} .
$$


But, uniqueness fails for the associated initial value problem.

This example is taken from M.G. Crandall and P.-L. Lions [5] and based on "pathological" situations for flows in $\mathbb{R}^{N}$ studied by A. Beck in [4]. We give nevertheless all the details of the construction for the convenience of the reader and in order to ensure that assumptions of Section 2 hold even in the case $X=B U C\left(\mathbb{R}^{N}\right)$.

Finally, in Section 6, we briefly present, as in [1], some necessary adjustements in order to extend the results of Section 3 to the case of two-parameters monotone families of operators $\left(T_{t, s}\right)_{t, s>0}$. We refer the reader to the book of W. Fleming and H.M. Soner [7] for developments about this kind of families in the context of viscosity solutions and optimal control and we just recall here that they are related to time-dependent equations

$$
\frac{\partial u}{\partial t}+F\left(t, x, u, D u, D^{2} u\right)=0 \quad \text { in }(0,+\infty) \times \mathbb{R}^{N},
$$

and that the semigroup property is replaced by

$$
T_{t, r}=T_{t, s} \circ T_{s, r} \quad \text { for all } t \geqslant s \geqslant r \geqslant 0 \text { and } T_{t, t}=I d_{X} .
$$

\section{Notations and assumptions on the semigroup}

We first describe the functional spaces we use througout this work and the related notations.

In the sequel, $X$ will denote a subspace of $\mathcal{C}\left(\mathbb{R}^{N}\right)$ satisfying the following conditions.

(H1) $X$ contains $\mathcal{D}\left(\mathbb{R}^{N}\right)$, the space of $\mathcal{C}^{\infty}$-functions with compact support in $\mathbb{R}^{N}$.

(H2) For every $f \in X$ and $y \in \mathbb{R}^{N}$, the function $x \mapsto f(x+y)$ belongs to $X$.

(H3) For every $u \in X$, there exists $g \in C_{X}^{\infty}\left(\mathbb{R}^{N}\right)$ such that $u \leqslant g$, where $C_{X}^{\infty}\left(\mathbb{R}^{N}\right)=$ $\mathcal{C}^{\infty}\left(\mathbb{R}^{N}\right) \cap X$.

It is worth noticing that most of the classical subspaces of $\mathcal{C}\left(\mathbb{R}^{N}\right)$ used in nonlinear analysis satisfy this three assumptions: $\mathcal{C}\left(\mathbb{R}^{N}\right), B U C\left(\mathbb{R}^{N}\right), W^{1, \infty}\left(\mathbb{R}^{N}\right)$, bounded continuous functions, uniformly continuous functions, continuous functions with growth conditions at infinity... etc.

We give now the assumptions we use throughout this work on the family of mappings $\left(T_{t}\right)_{t \geqslant 0}$ defined from $X$ into $X$ and make some comments about it. They are all more or less slightly weak versions of these used in [1] thus we use the same terminology.

[Causality]. -

$$
T_{t+s}=T_{t} \circ T_{s} \quad \text { for all } t, s \geqslant 0 \quad \text { and } \quad T_{0}=I d_{X} .
$$

[Monotonicity]. - For all $f, g \in X$

$$
f \leqslant g \Rightarrow T_{t}[f] \leqslant T_{t}[g] \text { for all } t \geqslant 0 .
$$

[Continuity]. - For every $u_{0} \in X$, the function $(t, x) \mapsto T_{t}\left[u_{0}\right](x)$ is continuous and for all $b>a \geqslant 0$ there exists $f_{a, b, u_{0}} \in C_{X}^{\infty}\left(\mathbb{R}^{N}\right)$ such that

$$
\left|T_{t}\left[u_{0}\right]\right| \leqslant f_{a, b, u_{0}} \quad \text { for all } t \in[a, b] .
$$


This last assumption may appear as being unusual and even restrictive. But, if $X$ is for example defined by growth conditions at infinity, it may be equiped with a norm. The assumption can be seen in this case as a relaxed version of the classical continuity of $t \mapsto T_{t}[f]$ in the norm sense.

[Locality]. - For every $f, g \in C_{X}^{\infty}\left(\mathbb{R}^{N}\right)$ and for any fixed $x$ in $\mathbb{R}^{N}$, if $f \equiv g$ on some $B(x, r)$ then

$$
T_{t}[f](x)-T_{t}[g](x)=\mathrm{o}(t) \quad \text { as } t \rightarrow 0^{+} .
$$

In order to state the next assumption, we introduce some particular subsets of $\mathcal{D}\left(\mathbb{R}^{N}\right)$ already used in [1]. If $d=\left(d_{n}\right)_{n \in \mathbb{N}}$ is a sequence of positive numbers, we set

$$
Q_{d}=\left\{f \in \mathcal{D}\left(\mathbb{R}^{N}\right),\left\|D^{\alpha} f\right\| \leqslant d_{n} \text { for } \alpha \in \mathbb{N}^{p} \text { with }|\alpha| \leqslant n\right\}
$$

where $|\alpha|=\alpha_{1}+\alpha_{2}+\cdots+\alpha_{p}$ if $\alpha=\left(\alpha_{1}, \ldots, \alpha_{p}\right) \in \mathbb{N}^{p}$.

The assumption (H1) together with the vector spaces structure of $X$ allows us to formulate the following assumption.

[Regularity]. - For any sequence of positive numbers $d$, for any compact subset $K \subset$ $\mathbb{R}^{N}$ and for every $f \in C_{X}^{\infty}\left(\mathbb{R}^{N}\right)$, there exists a positive function $m_{K, f, d}(\cdot): \mathbb{R}^{+} \mapsto \mathbb{R}^{+}$ with $m_{K, f, d}\left(0^{+}\right)=0$ such that

$$
\left|T_{t}[f+\lambda g](x)-T_{t}[f](x)-\lambda g(x)\right| \leqslant m_{K, f, d}(\lambda) t
$$

for any $(x, g) \in K \times Q_{d}$ and any $\lambda, t \geqslant 0$.

Compared to [1], and if we restrict ourselves to the $B U C$-framework, it is the assumption which is the most relaxed. More precisely, we use only $g$ with compact support and do not specify the dependence in $f$ for $m_{K, f, d}$. Moreover, $m_{K, f, d}$ can be an arbitrary modulus and not only a linear one.

If $x \in \mathbb{R}^{N}$, we denote by $\tau_{x}$ the translation operator on $\mathcal{C}\left(\mathbb{R}^{N}\right)$ defined by

$$
\tau_{x} \cdot f(y)=f(x+y)
$$

for $f \in \mathcal{C}\left(\mathbb{R}^{N}\right)$. Since $\tau_{x} \cdot X=X$ from (H2), we also denote by $\tau_{x}$ the restriction of $\tau_{x}$ to $X$.

[Translation]. - For any compact subset $K \subset \mathbb{R}^{N}$ and every $f \in \mathcal{D}\left(\mathbb{R}^{N}\right)$, there exists a function $n_{K, f}(\cdot): \mathbb{R}^{+} \rightarrow \mathbb{R}^{+}$with $n_{K, f}\left(0^{+}\right)=0$ such that

$$
\left|\tau_{x} \cdot T_{t}[f](y)-T_{t}\left(\tau_{x} \cdot f\right)(y)\right| \leqslant n_{K, f}(|x|) t
$$

for any $y \in K, t \geqslant 0$.

We recall that in [1], the semigroup $\left(T_{t}\right)_{t}$ was supposed to be invariant by translation i.e. $\tau_{x} \cdot T_{t}[f]=T_{t}\left(\tau_{x} \cdot f\right)$ for any $f \in X$ and $x \in \mathbb{R}^{N}$ and to take in account semigroups which do not satisfy such invariance property is also a main contribution of this article. 


\section{Generation of the parabolic P.D.E}

The main result is the:

THEOREM 3.1. - Let X be a subspace of $\mathcal{C}\left(\mathbb{R}^{N}\right)$ for which $(\mathrm{H} 1),(\mathrm{H} 2)$ and $(\mathrm{H} 3)$ hold. Let $\left(T_{t}\right)_{t \geqslant 0}$ a family of mappings from $X$ into $X$ satisfying [Causality], [Monotonicity], [Continuity], [Locality], [Regularity], and [Translation]. Then there exists a continuous function $F$ defined on $\mathbb{R}^{N} \times \mathbb{R} \times \mathbb{R}^{N} \times \mathcal{S}(N)$ such that (3) holds for any $f \in C_{X}^{\infty}\left(\mathbb{R}^{N}\right)$ and $x \in \mathbb{R}^{N}$. Moreover, $F$ satisfies the ellipticity condition (2) and the function $u(t, x):=$ $T_{t}\left[u_{0}\right](x)$ is a continuous viscosity solution of (1) for every initial data $u_{0} \in X$.

Proof. - For any $f \in C_{X}^{\infty}\left(\mathbb{R}^{N}\right)$ and $t>0$, we set

$$
\delta_{t}[f]=\frac{T_{t}[f]-f}{t} .
$$

In [1], in order to prove the existence of the infinitesimal generator, the main step was to show that, for $t$ small enough, $\left(\delta_{t}[f]\right)_{t \geqslant 0}$ was a Cauchy sequence in $B U C\left(\mathbb{R}^{N}\right)$. A rather difficut and technical task where [Regularity] together with the contraction property of $\left(T_{t}\right)_{t \geqslant 0}$ in $B U C\left(\mathbb{R}^{N}\right)$ were playing the main roles.

Here the key idea is to avoid this step by introducing and studying the mappings $\bar{A}$ and $\underline{A}$ defined on $C_{X}^{\infty}\left(\mathbb{R}^{N}\right)$ by setting

$$
\bar{A}[f](x)=\limsup _{t \rightarrow 0^{+}} \delta_{t}[f](x)
$$

and

$$
\underline{A}[f](x)=\liminf _{t \rightarrow 0^{+}} \delta_{t}[f](x) .
$$

The functions $\bar{A}$ and $\underline{A}$ can be seen respectively as the "upper infinitesimal generator" and the "lower infinitesimal generator" for the semigroup. The following lemma shows that they are well-defined.

LEMMA 3.1 (Boundedness of the upper and lower infinitesimal generator). - For any $x \in \mathbb{R}^{N}$ and $f \in C_{X}^{\infty}\left(\mathbb{R}^{N}\right), \bar{A}[f](x)$ and $\underline{A}[f](x)$ are finite.

The proof of Lemma 3.1 is based on the following technical result whose proof is postponed to Appendix A. We recall that $\mathcal{P}^{2,+}[u]\left(t_{0}, x_{0}\right)$ and $\mathcal{P}^{2,-}[u]\left(t_{0}, x_{0}\right)$ denote classically (see, for example, [6]) the second order parabolic semi-jets of the real-valued function $u$ at $\left(t_{0}, x_{0}\right)$.

Lemma 3.2. - Let $u_{0} \in X$ and $u(t, x):=T_{t}\left[u_{0}\right](x)$. If $(a, p, M) \in \mathcal{P}^{2,+}[u]\left(t_{0}, x_{0}\right)$ (respectively $\left.\mathcal{P}^{2,-}[u]\left(t_{0}, x_{0}\right)\right)$ then there exists a function $\tilde{\phi}:\left(t_{0}-a, t_{0}+a\right) \times \mathbb{R}^{N} \mapsto \mathbb{R}$ such that $\tilde{\phi}(t, \cdot) \in C_{X}^{\infty}\left(\mathbb{R}^{N}\right)$ for any $t \in\left(t_{0}-a, t_{0}+a\right)$ with

$$
\left(\tilde{\phi}\left(t_{0}, x_{0}\right), \frac{\partial \tilde{\phi}}{\partial t}\left(t_{0}, x_{0}\right), D \tilde{\phi}\left(t_{0}, x_{0}\right), D^{2} \tilde{\phi}\left(t_{0}, x_{0}\right)\right)=\left(u\left(t_{0}, x_{0}\right), a, p, M\right)
$$


and

$$
\bar{A}\left[\tilde{\phi}\left(t_{0}, \cdot\right)\right]\left(x_{0}\right) \geqslant \underline{A}\left[\tilde{\phi}\left(t_{0}, \cdot\right)\right]\left(x_{0}\right) \geqslant \frac{\partial \tilde{\phi}}{\partial t}\left(t_{0}, x_{0}\right)
$$

(respectively $\left.\frac{\partial \tilde{\phi}}{\partial t}\left(t_{0}, x_{0}\right) \leqslant \underline{A}\left[\tilde{\phi}\left(t_{0}, \cdot\right)\right]\left(x_{0}\right) \leqslant \bar{A}\left[\tilde{\phi}\left(t_{0}, \cdot\right)\right]\left(x_{0}\right)\right)$.

It is worth noticing that, a priori, the previous lemma does not say that the lim sup or liminf are finite.

Proof of Lemma 3.1. - 1) The continuity of $u(t, x)=T_{t}[0](x)$ implies that there exists $\left(t_{1}, x_{1}\right) \in(0,+\infty) \times \mathbb{R}^{N}$ (respectively $\left(t_{2}, x_{2}\right) \in(0,+\infty) \times \mathbb{R}^{N}$ ) such that $\mathcal{P}^{2,+}[u]\left(t_{1}, x_{1}\right) \neq \emptyset$ (respectively $\mathcal{P}^{2,-}[u]\left(t_{2}, x_{2}\right) \neq \emptyset$ ). Using Lemma 3.2, we deduce the existence of a function $\phi_{1} \in C_{X}^{\infty}\left(\mathbb{R}^{N}\right)$ (respectively $\phi_{2} \in C_{X}^{\infty}\left(\mathbb{R}^{N}\right)$ ) such that

$$
\underline{A}\left[\phi_{1}\left(t_{1}, \cdot\right)\right]\left(x_{1}\right) \geqslant \frac{\partial \phi_{1}}{\partial t}\left(t_{1}, x_{1}\right)
$$

and

$$
\bar{A}\left[\phi_{2}\left(t_{2}, \cdot\right)\right]\left(x_{2}\right) \leqslant \frac{\partial \phi_{2}}{\partial t}\left(t_{2}, x_{2}\right) .
$$

2) Using a standard truncation argument together with [Locality], we get the same inequalities for every $\Phi_{1}, \Phi_{2} \in \mathcal{D}\left(\mathbb{R}^{N}\right)$ such that $\Phi_{1} \equiv \phi_{1}\left(t_{1}, \cdot\right)$ (respectively $\Phi_{2} \equiv$ $\phi_{2}\left(t_{2}, \cdot\right)$ ) on some neighbourhood of $x_{1}$ (respectively $x_{2}$ ).

3) Using [Regularity] with $f=0, g=\Phi_{i}$ and $\lambda=1$, we get, for $i=1,2$

$$
\left|\delta_{t}[0]\left(x_{i}\right)-\delta_{t}\left[\Phi_{i}\right]\left(x_{i}\right)\right| \leqslant m_{x_{i}, \Phi_{i}}(1)
$$

we deduce from (6), together with (4) and (5) that

$$
\underline{A}[0]\left(x_{1}\right) \geqslant \frac{\partial \phi_{1}}{\partial t}\left(t_{1}, x_{1}\right)-m_{x_{1}, \Phi_{1}}(1)=C_{1}
$$

and

$$
\bar{A}[0]\left(x_{2}\right) \leqslant \frac{\partial \phi_{2}}{\partial t}\left(t_{2}, x_{2}\right)+m_{x_{2}, \Phi_{2}}(1)=C_{2} .
$$

4) Writing [Translation] for $f \equiv 0$ gives for any $t>0$ and $y \in \mathbb{R}^{N}$

$$
\left|\delta_{t}[0]\left(x_{i}+y\right)-\delta_{t}[0]\left(x_{i}\right)\right| \leqslant n\left(x_{i}, y\right) \text { for } i=1,2
$$

and together with inequalities (7) and (8) this implies that, for any $x \in \mathbb{R}^{N}, \bar{A}[0](x)$ and $\underline{A}[0](x)$ are finite.

5) We consider now $g \in \mathcal{D}\left(\mathbb{R}^{N}\right)$ and $x \in \mathbb{R}^{N}$. Using [Regularity] as in step 3) and the previous result, we get a bound for $\bar{A}[g](x)$ and $\underline{A}[g](x)$. We conclude that the same property holds for $g \in C_{X}^{\infty}\left(\mathbb{R}^{N}\right)$ using again [Locality] together with a standard truncation arguments.

Now we turn to further properties of $\bar{A}$ and $\underline{A}$. 
LEMMA 3.3 (Structure of the upper and lower infinitesimal generator). - There exist two functions $\underline{F}, \bar{F} \in \mathcal{C}\left(\mathbb{R}^{N} \times \mathbb{R} \times \mathbb{R}^{N} \times \mathcal{S}(N)\right)$ satisfying (2) such that, for every $f \in C_{X}^{\infty}\left(\mathbb{R}^{N}\right)$ and $x \in \mathbb{R}^{N}$,

$$
\bar{A}[f](x)=-\bar{F}\left(x, f(x), D f(x), D^{2} f(x)\right)
$$

and

$$
\underline{A}[f](x)=-\underline{F}\left(x, f(x), D f(x), D^{2} f(x)\right) .
$$

We postpone the proof of this lemma to the end of the present section and observe that in view of them, the notion of viscosity solution is well-defined for the parabolic problems associated to $\bar{F}$ and $\underline{F}$. The following lemma is then nothing that a direct consequence of Lemma 3.2 together with the definition of continuous viscosity solutions.

LEMMA 3.4 (Semigroup, infinitesimal generators and parabolic equations). - Let $u_{0} \in X$. Then the function $u(t, x):=T_{t}\left[u_{0}\right](x)$ is a continuous viscosity solution of

$$
\frac{\partial u}{\partial t}+\underline{F}\left(x, u, D u, D^{2} u\right)=0 \quad \text { in }(0,+\infty) \times \mathbb{R}^{N}
$$

and

$$
\frac{\partial u}{\partial t}+\bar{F}\left(x, u, D u, D^{2} u\right)=0 \quad \text { in }(0,+\infty) \times \mathbb{R}^{N} .
$$

We have now, in order to complete the proof of Theorem 3.1, to show that $\bar{F}=\underline{F}$.

LEMMA 3.5 (Existence of the infinitesimal generator). - For any $(x, r, p, M) \in$ $\mathbb{R}^{N} \times \mathbb{R} \times \mathbb{R}^{N} \times \mathcal{S}(N)$, we have

$$
\underline{F}(x, r, p, M)=\bar{F}(x, r, p, M) .
$$

In particular, if we set $F:=\bar{F}=\underline{F}$ then, for any $f \in C_{X}^{\infty}\left(\mathbb{R}^{N}\right)$, we have

$$
\lim _{t \rightarrow 0^{+}} \frac{T_{t}[f](x)-f(x)}{t}=-F\left(x, f(x), D f(x), D^{2} f(x)\right) .
$$

The proof of Theorem 3.1 is indeed complete since it shows that there exists a function $F:=\underline{F}=\bar{F}$ defined on $\mathbb{R}^{N} \times \mathbb{R} \times \mathbb{R}^{N} \times \mathcal{S}(N)$ such that (3) holds for any $f \in C_{X}^{\infty}\left(\mathbb{R}^{N}\right)$ and $x \in \mathbb{R}^{N}$. The function $F$ is continuous and elliptic since, by Lemma 3.3, $\underline{F}$ an $\bar{F}$ are continuous and elliptic and from Lemma 3.4, the function $u(t, x):=T_{t}\left[u_{0}\right](x)$ is a continuous viscosity solution of (1) for every initial data $u_{0} \in X$.

It remains to prove Lemmas 3.5 and 3.3.

Proof of Lemma 3.5. - To any $(x, r, p, M) \in \mathbb{R}^{N} \times \mathbb{R} \times \mathbb{R}^{N} \times \mathcal{S}(N)$, we associate the function $f_{x, r, p, M}$ defined for $y \in \mathbb{R}^{N}$ by

$$
f_{x, r, p, M}(y)=\left(r+\langle p, y-x\rangle+\frac{1}{2}\langle M \cdot(y-x),(y-x)\rangle\right) v(y-x),
$$


where $v$ is a fixed function in $\mathcal{D}\left(\mathbb{R}^{N}\right)$ with a compact support in $B(0,1)$ such that $0 \leqslant v \leqslant 1$ and $v \equiv 1$ on $B(0,1 / 2)$.

In fact, because of Lemma 3.3, we have to prove that

$$
\limsup _{h \rightarrow 0^{+}} \frac{T_{h}[f](x)-f(x)}{h} \leqslant-\underline{F}(x, f(x), p, M),
$$

where $f=f_{x, r, p, M}$. We set $u(t, y):=T_{t}[f][y]$ and proceed in several steps.

1) We introduce the family of functions $\left(W_{\eta, \delta}\right)_{\eta, \delta>0}$ defined in $0,(\infty) \times \mathbb{R}^{N}$ by

$$
W_{\eta, \delta}(t, y)=f(y)+\eta|y-x|^{2}+t[\underline{F}(x, f(x), p, M)+\delta]
$$

and claim that for every $\delta>0$, there exists $\eta, T, r>0$ such that

$$
u(t, y) \leqslant W_{\eta, \delta}(t, y) \quad \text { on }[0, T] \times \bar{B}(x, r)=\bar{\Omega}_{T, r} .
$$

To prove our claim, we set $\phi_{\eta, \delta}=u-W_{\eta, \delta}$ and

$$
M_{\eta, \delta, T, r}=\sup _{\bar{\Omega}_{T, r}} \phi_{\eta, \delta} .
$$

We have to show that $M_{\eta, \delta, T, r} \leqslant 0$ for a suitable choice of parameters.

2) To do so, we first remark that, since $u$ and $\underline{F}$ are continuous, for every $\delta>0$, there exists $\eta, T, r>0$ such that

$$
\frac{\partial W_{\eta, \delta}}{\partial t}>-\underline{F}\left(y, u(t, y), D W_{\eta, \delta}, D^{2} W_{\eta, \delta}\right) \quad \text { on } \bar{\Omega}_{T, r} .
$$

3) It is clear that (10) holds on $\{0\} \times B\left(x_{0}, r\right)$ for every $\eta, r>0$ with a strict inequality for $x \neq x_{0}$ and thus on $\{0\} \times \partial B\left(x_{0}, r\right)$. Using the continuity of $u$ and the previous observation, we conclude that for every $\eta, r$ there exists $T(\eta, r)>0$ such that (10) holds on the lateral boundary $[0, T(\eta, r)] \times \partial B\left(x_{0}, r\right)$.

4) We choose the parameter $\eta, T, r$ according to point 2) and 3) above and we assume by contradiction that $M_{\eta, \delta, T, r}>0$. Then necessarily, this maximum is achieved at an interior point $(\bar{t}, \bar{x}) \in \Omega_{T, r}$ or for $\bar{t}=T$. Since $u$ is a viscosity solution of the initial value problem (1) with $F=\underline{F}$ and since $W_{\eta, \delta} \in C^{2}\left([0,+\infty) \times \mathbb{R}^{N}\right)$, it follows, by definition, that

$$
\frac{\partial W_{\eta, \delta}}{\partial t}(\bar{t}, \bar{y}) \leqslant-\underline{F}\left(\bar{y}, u(\bar{t}, \bar{y}), D_{\eta, \delta}(\bar{t}, \bar{y}), D^{2} W_{\eta, \delta}(\bar{t}, \bar{y})\right)
$$

even if $\bar{t}=T$ (see [3]). But, this inequality contradicts the property (11) above and therefore (10) holds.

5) We conclude by writing (10) at $y=x$. For $t$ sufficiently small

$$
\frac{T_{t}[f](x)-f(x)}{t} \leqslant\left[-\underline{F}\left(x, f(x), D f(x), D^{2} f(x)\right)+\delta\right] .
$$

Taking the lim sup for $t \rightarrow 0^{+}$and then letting $\delta \rightarrow 0^{+}$, we complete the proof of Lemma 3.5 and also the proof of Theorem 3.1. 
We conclude this section by giving the proof of Lemma 3.3. We provide the proof for $\bar{A}$, the proof for $\underline{A}$ being essentially the same with straightforward adaptations. We follow the idea of [1].

1) To obtain that $\bar{A}[f](x)=\bar{F}\left(x, f(x), D f(x), D^{2} f(x)\right)$, we take $x \in \mathbb{R}^{N}$ and consider two functions $f, g \in C_{X}^{\infty}\left(\mathbb{R}^{N}\right)$ with $D^{\alpha} f(x)=D^{\alpha} g(x)$ for $|\alpha| \leqslant 2$. We introduce a function $f_{\varepsilon}$ by setting

$$
f_{\varepsilon}(y)=f(y)+\varepsilon|y-x|^{2} \cdot v(y-x)
$$

recalling that $v$ is the smooth truncation defined in in the proof of Lemma 3.5.

By Taylor's formula, $f_{\varepsilon}$ is greater than $g$ on $B\left(x, r_{\varepsilon}\right)$ for a suitable $r_{\varepsilon}$. But in order to use [Monotonicity], we need an inequality in the whole space. To this end, we use the function $v_{\varepsilon}(y)=v\left((y-x) / r_{\varepsilon}\right)$ and define

$$
\bar{f}_{\varepsilon}=f_{\varepsilon} v_{\varepsilon}, \quad \bar{g}_{\varepsilon}=g v_{\varepsilon} .
$$

We clearly have

$$
\bar{f}_{\varepsilon} \geqslant \bar{g}_{\varepsilon}
$$

and $\bar{f}_{\varepsilon}, \bar{g}_{\varepsilon} \in C_{X}^{\infty}\left(\mathbb{R}^{N}\right)$ by $(\mathrm{H} 1)$.

2) Applying [Monotonicity] to the previous inequality we get

$$
T_{t}\left(\bar{f}_{\varepsilon}\right) \geqslant T_{t}\left(\bar{g}_{\varepsilon}\right)
$$

Since $\bar{f}_{\varepsilon}(x)=f_{\varepsilon}(x)=f(x)=g(x)$, we have

$$
T_{t}\left(\bar{f}_{\varepsilon}\right)(x)-\bar{f}_{\varepsilon}(x) \geqslant T_{t}\left(\bar{g}_{\varepsilon}\right)(x)-g(x) .
$$

Then, since $\bar{f}_{\varepsilon} \equiv f_{\varepsilon}$ and $g \equiv \bar{g}_{\varepsilon}$ on $B\left(x, r_{\varepsilon} / 2\right)$, we can use [Locality] to obtain

$$
T_{t}\left(f_{\varepsilon}\right)(x)-f_{\varepsilon}(x)+\mathrm{o}(\varepsilon, t) \geqslant T_{t}(g)(x)-g(x) .
$$

Using now [Regularity] with $f \in C_{X}^{\infty}\left(\mathbb{R}^{N}\right), w=|\cdot-x|^{2} v(\cdot-x) \in \mathcal{D}\left(\mathbb{R}^{N}\right)$ and $\lambda=\varepsilon$ we get

$$
T_{t}(f)(x)-f(x)+\mathrm{o}(\varepsilon, t) \geqslant T_{t}(g)(x)-g(x)-m_{x, f, w}(\varepsilon) t .
$$

Dividing by $t$ and taking the lim sup for $t \rightarrow 0^{+}$, and then letting $\varepsilon \rightarrow 0$ we obtain

$$
\bar{A}[f](x) \geqslant \bar{A}[g](x) .
$$

Since the previous computations are symmetric in $f$ and $g$, we get the equality.

3) Here and therein $(x, r, p, M)$ will ever denote an arbitrary element of $\mathbb{R}^{N} \times \mathbb{R} \times$ $\mathbb{R}^{N} \times \mathcal{S}(N)$ and $f_{x, r, p, M} \in \mathcal{D}\left(\mathbb{R}^{N}\right)$ the function defined by (9). We define the function $\bar{F}$ on $\mathbb{R}^{N} \times \mathbb{R} \times \mathbb{R}^{N} \times \mathcal{S}(N)$ by setting

$$
-\bar{F}(x, r, p, M)=\bar{A}\left[f_{x, r, p, M}\right](x)
$$


and thanks to the previous point we have for any $f \in C_{X}^{\infty}\left(\mathbb{R}^{N}\right)$ and $x \in \mathbb{R}^{N}$

$$
\bar{A}[f](x)=-\bar{F}\left(x, f(x), D f(x), D^{2} f(x)\right) .
$$

The ellipticity of $\bar{F}$ is then a direct consequence of [Monotonicity] together with the obvious following fact

$$
N \geqslant M \Rightarrow f_{x, r, p, N} \geqslant f_{x, r, p, M}
$$

4) To complete the proof of the proposition, we need to see that the function $\bar{F}$ is continuous.

Let $\left(x, r_{0}, p_{0}, M_{0}\right) \in \mathbb{R}^{N} \times \mathbb{R} \times \mathbb{R}^{N} \times \mathcal{S}(N)$ and set $f_{0}=f_{x_{0}, r_{0}, p_{0}, M_{0}}$. We have, using [Translation], that

$$
\begin{aligned}
\left|T_{t}\left[f_{0}\right]\left(x_{0}\right)-T_{t}\left[\tau_{-x} \cdot f_{0}\right]\left(x_{0}+x\right)\right| & =\left|\tau_{-x} \cdot T_{t}\left[f_{0}\right]\left(x_{0}+x\right)-T_{t}\left[\tau_{-x} f_{0}\right]\left(x_{0}+x\right)\right| \\
& \leqslant n_{x_{0}, f_{0}}(|x|) t .
\end{aligned}
$$

and thus,

$$
\left|\bar{F}\left(x_{0}, r_{0}, p_{0}, M_{0}\right)-\bar{F}\left(x_{0}+x, r_{0}, p_{0}, M_{0}\right)\right| \leqslant n_{x_{0}, f_{0}}(|x|) .
$$

Moreover, for any $R>0$ and any positive sequence $d$, we can rewrite [Regularity] under the form

$$
\left|\bar{A}\left[f_{0}+\varepsilon g\right]\left(x_{0}+x\right)-\bar{A}\left[f_{0}\right]\left(x_{0}+x\right)\right| \leqslant m_{x_{0}, R, f_{0}, d}(\varepsilon)
$$

for any $(x, g) \in B(0, R) \times Q_{d}$. We apply the previous inequality with

$$
g_{\varepsilon}=f_{x_{0}+x, r / \varepsilon, p / \varepsilon, M / \varepsilon}
$$

noticing that the $g_{\varepsilon}$ are in a same $Q_{d}$ for $|r|,|p|,|M| \leqslant \varepsilon$ and get that there exists an $R>0$ such that

$$
\left|\bar{F}\left(x_{0}+x, r_{0}, p_{0}, M_{0}\right)-\bar{F}\left(x_{0}+x, r_{0}+r, p_{0}+p, M_{0}+M\right)\right| \leqslant m_{x_{0}, R, f_{0}, d}(\varepsilon)
$$

for any $|x|,|r|,|p|,|M| \leqslant \varepsilon$.

From (13) and (14), we complete the proof of the continuity.

Remark 3.1. - We want to point out that our proof works if [Regularity] is stated only for functions $f$ appearing in [Continuity] or which belong to $\mathcal{D}\left(\mathbb{R}^{N}\right)$ instead of $f \in C_{X}^{\infty}\left(\mathbb{R}^{N}\right)$. In particular, if $X$ is a subspace of bounded functions as in [1], it suffices to write the assumption only for $f \in C_{b}^{\infty}\left(\mathbb{R}^{N}\right)$.

\section{Consequences of additional properties for the semigroup}

We start with elementary facts. The proofs of these facts are straightforward using Theorem 3.1, so we will omit them.

Proposition 4.1. - Let $F$ be the function which appears in Theorem 3.1. 
(1) If $T_{t}$ is linear for any $t$, there exists continuous functions $a, b, c$ from $\mathbb{R}^{N}$ to $\mathcal{S}^{+}(N), \mathbb{R}^{N}$ and $\mathbb{R}$ respectively, such that

$$
F(x, r, p, M)=-\operatorname{Tr}[a(x) M]+\langle b(x), p\rangle+c(x) r,
$$

where $\mathcal{S}^{+}(N)$ denote the space of the positive symmetric matrices.

(2) If $T_{t}[f+C]=T_{t}[f]+C$ for any $f \in X$ and $C \in \mathbb{R}$ then $F$ is independent of $r$.

(3) If $\tau_{x}\left[T_{t}(f)\right]=T_{t}\left[\tau_{x}(f)\right]$ for all $(t, x) \in[0,+\infty) \times \mathbb{R}^{N}$ and $f \in \mathcal{D}\left(\mathbb{R}^{N}\right)$ then $F$ is independent of $x$.

It is well-known that, under suitable assumptions, first-order Hamilton-Jacobi equations satisfy properties of "finite speed of propagation" or "domain of dependence". Such properties were first proved by M.G. Crandall and P.-L. Lions in [5] (see also I. Ishii [9] or O. Ley [10] for a different proof).

We address now the question of the converse property: is a semigroup satisfying some domain of dependence-type property associated to a first-order equation? The answer is yes and a first result in this direction was first proved by P.-L. Lions in [12] under slightly stronger but less numerous assumptions than here.

We introduce the following formulation of a domain of dependence property for $\left(T_{t}\right)_{t} \geqslant 0$

[Strong-Locality]. - For any $f, g \in X$ and any compact subset $K \subset \mathbb{R}^{N}$ there exists a positive constant $L_{K, f, g}$ and $0<s<2$ such that

$$
f \leqslant g \text { in } B\left(x_{0}, R\right) \Rightarrow T_{t}[f]\left(x_{0}\right) \leqslant T_{t}[g]\left(x_{0}\right) \text { for } t \leqslant L_{K, f, g} R^{s}
$$

for any $x_{0} \in K$ and any $R>0$.

THEOREM 4.1. - Let $\left(T_{t}\right)_{t \geqslant 0}$ satisfy the assumptions of Theorem 3.1 where [Locality] and [Monotonicity] are replaced by [Strong-Locality]. There exists a function $F \in$ $C\left(\mathbb{R}^{N} \times \mathbb{R} \times \mathbb{R}^{N}\right)$ such that, for any $u_{0} \in X$, the function $(t, x) \mapsto T_{t}\left[u_{0}\right](x)$ is a viscosity solution of the Hamilton-Jacobi Equation associated to $F$, i.e.

$$
\frac{\partial u}{\partial t}+F(x, u, D u)=0 \quad \text { in }(0,+\infty) \times \mathbb{R}^{N}
$$

Proof of Theorem 4.1. - First it is clear that [Strong-Locality] implies [Monotonicity] and [Locality] and thus Theorem 3.1 holds. Therefore we have only to prove that

$$
F(x, r, p, A)=F(x, r, p, B) \quad \text { for any }(x, r, p, A, B) \in \mathbb{R}^{N} \times \mathbb{R} \times \mathbb{R}^{N} \times \mathcal{S}(N)^{2}
$$

To this end, we consider the functions $f_{x, r, p, A}$ and $f_{x, r, p, B}$ and $1 / 2>\eta>0$. We have

$$
f_{x, r, p, A} \leqslant f_{x, r, p, B}+\frac{|B-A|}{2} \eta^{2} v(\cdot-x) \quad \text { in } B(x, \eta)
$$

for $\eta$ sufficiently small, where $v$ is the smooth truncation function defined in the proof of Lemma 3.5. 
Using [Strong-Locality] and [Regularity], this implies

$$
T_{t}\left[f_{x, r, p, A}\right](x) \leqslant T_{t}\left[f_{x, r, p, B}\right](x)+\frac{|B-A|}{2} \eta^{2}+m_{x, r, p, A, B}\left(\eta^{2}\right) t \quad \text { for } t \leqslant L \eta^{s}
$$

with $L$ independent of $\eta$. Subtracting $r$, we use this inequality for $t=L \eta^{s}$, divide by $L \eta^{s}$ and finally we let $\eta \rightarrow 0^{+}$. We obtain

$$
-F(x, r, p, A) \leqslant-F(x, r, p, B)
$$

and we conclude by the symmetry of $A$ and $B$ in the previous arguments.

Remark 4.1.- More regularity on $F$ occurs with respect to $p$ if we add more restrictions on the term $L R^{s}$ in [Strong-Locality]. For example, if $L$ is independent of $(x, f, g)$ and $s=1$, the nonlinearity $F$ is Lipschitz continuous in $p$ uniformly with respect to the other variables.

\section{On the uniquenes for the associated p.d.e}

We will show in this section that uniqueness may fail in general for viscosity solutions of the partial differential equation associated to a semigroup which satisfies only assumptions of Theorem 3.1 or Theorem 4.1 even if $X=B U C\left(\mathbb{R}^{N}\right)$.

Our example is entirely taken from [5] where it was used by Crandall and Lions as a counter-example to uniqueness for viscosity solution of a transport equation when the natural assumptions are not satisfied. This is related to the non-uniqueness for flows of the associated dynamic studied by Beck in [4].

LEMMA 5.1. - There exist two different continuously differentiable homeomorphism $f, h$ on $\mathbb{R}$ such that

$$
f^{\prime}\left(f^{-1}(x)\right)=h^{\prime}\left(h^{-1}(x)\right) \quad \text { for any } x \in \mathbb{R} .
$$

In addition, one can impose that $f, f^{-1}, h, h^{-1}$ are uniformly continuous on $\mathbb{R}$ and $f^{\prime}$, $f^{\prime \prime}, f^{\prime \prime \prime}$ bounded.

Proof. - We follow the ideas of [4] and just introduce slightly specifications in order to ensure that the assumptions of Section 2 hold.

1) We consider a Cantor set $K \subset[0,1]$ with a strictly positive Lebesgue measure and a smooth function $g$ such that $0 \leqslant g \leqslant 1, g(x)=0$ if and only if $x \in K, g^{\prime}, g^{\prime \prime}$ bounded on $\mathbb{R}$ and $g \geqslant \eta>0$ on $(-\infty,-1] \cup[2,+\infty)$. Then we define a function $f$ by setting

$$
f(x)=\int_{0}^{x} g(\tau) d \tau .
$$

Clearly, $f$ is a Lipschitz continuous differentiable homeomorphism from $\mathbb{R}$ to $\mathbb{R}$. Moreover the last requirement on $g$ ensures that $f^{-1}$ is Lipschitz continuous on 
$\left(-\infty, f^{-1}(-1)\right] \cup\left[f^{-1}(2),+\infty\right)$ and thus is uniformly continuous on $\mathbb{R}$ since it is continuous on $\mathbb{R}$.

2) We construct an other differentiable homeomorphism $h$ by setting successively

$$
\alpha(x)=x+\mu(K \cap[0, x]),
$$

where $\mu$ denote the Lebesgue measure on $\mathbb{R}$ and then

$$
h=f \circ \alpha^{-1} .
$$

The function $\alpha$ is strictly increasing continuous and thus is an homeomorphism from $\mathbb{R}$ to $\mathbb{R}$. Moreover it is a Lipschitz continuous function on $\mathbb{R}$. Finally we have the following inequality

$$
\alpha\left(y_{2}\right)-\alpha\left(y_{1}\right) \geqslant y_{2}-y_{1} \quad \text { for any } y_{2} \geqslant y_{1}
$$

which ensures that $\alpha^{-1}$ is also Lipschitz continuous.

It follows finally that $h$ and $h^{-1}$ are also uniformly continuous.

3) We check the property (16).

(a) We first consider $x \in \mathbb{R}$ such that $f^{-1}(x) \notin K$. We want to check that $h$ is differentiable at $h^{-1}(x)$. But, since $f^{-1}(x) \notin K$, there is a neighborhood of $f^{-1}(x)$ on which $\alpha$ is nothing but $I d_{\mathbb{R}}+c$ for some constant $c$, and therefore $\alpha$ is differentiable at $f^{-1}(x)$ with derivative 1 .

We deduce from this fact that $\alpha^{-1}$ is differentiable at $\alpha\left(f^{-1}(x)\right)=h^{-1}(x)$ with derivative 1 and then that $h$ is differentiable at $h^{-1}(x)$ with

$$
h^{\prime}\left(h^{-1}(x)\right)=1 \times f^{\prime}\left(\alpha^{-1}\left(h^{-1}(x)\right)=f^{\prime}\left(f^{-1}(x)\right) .\right.
$$

(b) Let $x \in \mathbb{R}$ such that $y=f^{-1}(x) \in K$. We have

$$
\begin{aligned}
h\left(h^{-1}(x)\right)-h(z) & =f\left[\alpha^{-1}\left(h^{-1}(x)\right)\right]-f\left[\alpha^{-1}(z)\right] \\
& \leqslant \sup _{I_{z}}\left|f^{\prime}\right| \cdot K_{\alpha^{-1}} \cdot\left|\left(h^{-1}(x)\right)-z\right|,
\end{aligned}
$$

where $K_{\alpha^{-1}}$ denote the Lipschitz constant for $\alpha^{-1}$ and $I_{X}=\left[\alpha^{-1}\left(h^{-1}(x)\right), \alpha^{-1}(z)\right]$. Since $f$ is $C^{1}$ and $f^{\prime}\left(\alpha^{-1}\left(h^{-1}(x)\right)\right)=f^{\prime}\left(f^{-1}(x)\right)=0$ recalling $f^{-1}(x) \in K$ we obtain dividing by $\left(h^{-1}(x)\right)-X$ and letting $X \rightarrow h^{-1}(x)$ that $h$ is differentiable at $h^{-1}(x)$ with

$$
h^{\prime}\left(h^{-1}(x)\right)=0=f^{\prime}\left(f^{-1}(x)\right) .
$$

Following [5] we now define two flows by setting $Y_{f}(t, x)=f\left(t+f^{-1}(x)\right)$ and $Y_{h}(t, x)=h\left(t+h^{-1}(x)\right)$. There flows are distinct and provide two semigroups on $B U C(R)$ by setting

$$
T_{t}^{f}\left[u_{0}\right](x)=u_{0}\left(Y_{f}(t, x)\right) \quad\left(\text { respectively } T_{t}^{h}\left[u_{0}\right](x)=u_{0}\left(Y_{h}(t, x)\right)\right)
$$

for every $u_{0} \in B U C(\mathbb{R})$. 
Proposition 5.1. - The linear semigroup $\left(T_{t}^{f}\right)_{t \geqslant 0}$ satisfies all the assumptions Theorem 4.1 with $X=B U C(\mathbb{R})$. Moreover $T_{t}^{f}$ and $T_{t}^{h}$ give both viscosity solutions of

$$
\frac{\partial u}{\partial t}+b(x) \cdot D u(t, x)=0 \quad \text { in }(0,+\infty) \times \mathbb{R},
$$

where $b(x)=f^{\prime}\left(f^{-1}(x)\right)=h^{\prime}\left(h^{-1}(x)\right)$.

Sketch of proof of Proposition 5.1. - It is easy to check that $\left(T_{t}^{f}\right)_{t \geqslant 0}$ (respectively $\left(T_{t}^{h}\right)_{t \geqslant 0}$ ) is defined on $B U C(\mathbb{R})$ using and that $f^{-1}$ (respectively $h^{-1}$ ) is uniformly continuous. Then, straightforward computations involving the linearity of the semigroups and the differentiability of $f$ and $h$ show that [Continuity] [Strong-Locality] and [Regularity] hold for $\left(T_{t}^{f}\right)_{t \geqslant 0}$ and $\left(T_{t}^{h}\right)_{t \geqslant 0}$.

Moreover $\left(T_{t}^{f}\right)_{t \geqslant 0}$ satisfies [Translation]. Indeed, for $u_{0} \in \mathcal{D}(\mathbb{R})$, we write

$$
\begin{aligned}
& \left|\tau_{y} \cdot T_{t}^{f}\left[u_{0}\right](x)-T_{t}^{f}\left[\tau_{y} \cdot u_{0}\right](x)\right| \\
& \quad=\left|u_{0}\left[f\left(-t+f^{-1}(x+y)\right)\right]-u_{0}\left[f\left(-t+f^{-1}(x)\right)+y\right]\right| \\
& \quad \leqslant\left\|D u_{0}\right\|_{\infty} \cdot\left|f\left(-t+f^{-1}(x+y)\right)-f\left(-t+t^{-1}(x)\right)-y\right| .
\end{aligned}
$$

But, using a Taylor's formula, we have

$$
\begin{aligned}
& \left|f\left(-t+f^{-1}(x+y)\right)-f\left(-t+f^{-1}(x)\right)-y\right| \\
& \quad \leqslant t|b(x+y)-b(x)|+t^{2} \int_{0}^{1} \frac{(1-h)^{2}}{2}\left|f^{\prime \prime}\left(f^{-1}(x+y)-h t\right)-f^{\prime \prime}\left(f^{-1}(x)-h t\right)\right| d h
\end{aligned}
$$

and we get that [Translation] holds for $\left(T_{t}^{f}\right)_{t \geqslant 0}$ using the boundedness of $f^{\prime \prime \prime}$ together with the uniform continuity of $b=f^{\prime} \circ f^{-1}$.

But it is worth mentioning that it is not clear that the semigroup $\left(T_{t}^{h}\right)_{t}$ satisfies the [Translation] property. Anyway, as in [5], $u_{f}(t, x):=T_{t}^{f}\left[u_{0}\right](x)$ and $u_{h}(t, x):=$ $T_{t}^{h}\left[u_{0}\right](x)$ are both continuous viscosity solutions of (19), this fact being also a consequence of Theorem 3.1 only for $u_{f}$.

This example shows in particular that, in general, uniqueness may fail for viscosity solutions of the initial value problems deduced from semigroups satisfying only the assumptions of Section 2.

\section{The case of two-parameters families}

We give briefly the generalization of Theorem 3.1 for a two-parameters family $\left(T_{t, s}\right)_{t \geqslant s \geqslant 0}$ from $X$ into $X$. As in [1], we use adaptations of the assumptions used for the one-parameter case.

[Causality]. -

$$
T_{t, r}=T_{t, s} \circ T_{s, r} \quad \text { for any } t \geqslant s \geqslant r \geqslant 0 \quad \text { and } \quad T_{t, t}=I d_{X} .
$$


[Monotonicity]. - For any $f, g \in X, t \geqslant h \geqslant 0$,

$$
f \geqslant g \Rightarrow T_{t, t-h}[f] \geqslant T_{t, t-h}[g]
$$

[Continuity]. - For any $u_{0} \in X, t \geqslant 0$, the function $u(h, x):=T_{t+h, t}\left[u_{0}\right](x)$ is continuous and for any $b \geqslant a \geqslant 0$, there exists $f_{u_{0}, a, b} \in C_{X}^{\infty}\left(\mathbb{R}^{N}\right)$ such that

$$
\left|T_{t+h, t}\left[u_{0}\right]\right| \leqslant f_{t, u_{0}, a, b} \text { for any } h \in[a, b] .
$$

[Locality]. - For any $f, g \in C_{X}^{\infty}\left(\mathbb{R}^{N}\right)$ and $x \in \mathbb{R}^{N}$, if $f \equiv g$ in $B(x, R)$ for some $R>0$ then

$$
T_{t, t-h}[f](x)-T_{t, t-h}[g](x)=\mathrm{o}(h) \quad \text { as } h \rightarrow 0^{+} .
$$

[Regularity]. - For any sequence of positive numbers $d$, any compact subset $K \subset$ $\mathbb{R}^{N} \times[0,+\infty)$ and every $f \in C_{X}^{\infty}\left(\mathbb{R}^{N}\right)$ there exists a positive function $m_{K, f, d}(\cdot)$ with $m_{K, d, f}\left(0^{+}\right)=0$ such that

$$
\left|T_{t, t-h}[f+\lambda g](x)-T_{t, t-h}[f](x)-\lambda g(x)\right| \leqslant m_{K, f, d}(\lambda) h
$$

for every $((x, t), g) \in K \times Q_{d}, \lambda \geqslant 0,0 \leqslant h \leqslant t$.

[Translation]. - For any compact subset $K \subset \mathbb{R}^{N} \times[0,+\infty)$ and any $f \in \mathcal{D}\left(\mathbb{R}^{N}\right)$ there exists a function $n_{K, f}(\cdot)$ with $n_{K, f}\left(0^{+}\right)=0$ such that

$$
\left|\tau_{y} \cdot T_{t, t-h}[f](x)-T_{t, t-h}\left(\tau_{y} \cdot f\right)(x)\right| \leqslant n_{K, f}(|y|) h
$$

for every $((x, t), f) \in K \times Q_{d}, y \in \mathbb{R}^{N}, 0 \leqslant h \leqslant t$.

In addition, we need as in [1] the following property:

[Stability]. - For every sequence of positive numbers $d$ and any compact subset $K \subset \mathbb{R}^{N}$, there exists a function $p_{K, d}(\cdot)$ with $p_{K, d}\left(0^{+}\right)=0$ such that

$$
\left|T_{t, t-h}[f](x)-T_{t^{\prime}, t^{\prime}-h}[f](x)\right| \leqslant p_{K, d}\left(\left|t-t^{\prime}\right|\right) h
$$

for any $(x, f) \in K \times Q_{d}, t, t^{\prime} \geqslant h \geqslant 0$.

We have then the:

THEOREM 6.1. - Let $\left(T_{t, s}\right)_{t \geqslant s \geqslant 0}$ a family of mappings defined from $X$ into $X$ where $X \subset C\left(\mathbb{R}^{N}\right)$, satisfying [Causality], [Monotonicity], [Continuity], [Locality], [Regularity], [Translation] and [Stability] and $X$ satisfies (H1)-(H3). There exists a continuous function $F$ on $[0,+\infty) \times \mathbb{R}^{N} \times \mathbb{R} \times \mathbb{R}^{N} \times \mathcal{S}(N)$ such that for any $f \in$ $C_{X}^{\infty}\left(\mathbb{R}^{N}\right), x \in \mathbb{R}^{N}$

$$
\lim _{h \rightarrow 0^{+}} \frac{T_{t, t-h}[f](x)-f(x)}{h}=A_{t}[f](x)=-F\left(t, x, f(x), D f(x), D^{2} f(x)\right) .
$$


Moreover, the function $F$ satisfies the ellipticity condition (2) and for any $u_{0} \in X$, the function $u(t, x):=T_{t, 0}\left[u_{0}\right](x)$ is a continuous viscosity solution of the Cauchy problem

$$
\begin{cases}\frac{\partial u}{\partial t}+F\left(t, x, u, D u, D^{2} u\right)=0 & \text { in }(0,+\infty) \times \mathbb{R}^{N}, \\ u(0, \cdot)=u_{0} & \text { in } \mathbb{R}^{N} .\end{cases}
$$

Proof. - First, we observe that for every fixed $t \geqslant 0$ the mappings $\left(T_{t, t-h}\right)_{t \geqslant h \geqslant 0}$ satisfy the same properties as $\left(T_{h}\right)_{h \geqslant 0}$ except [Causality]. Thus the proof is nothing else that a straigthforward adaptation of those we have given in the one parameter case. Therefore we do not mention every details and just give the essential adjustements.

Again, we define two time-dependent mappings by

$$
\limsup _{h \rightarrow 0^{+}} \frac{T_{t, t-h}[f](x)-f(x)}{h}=\limsup _{h \rightarrow 0^{+}} \delta_{t, t-h}[f](x)=\bar{A}_{t}[f](x)
$$

and

$$
\liminf _{h \rightarrow 0^{+}} \frac{T_{t, t-h}[f](x)-f(x)}{h}=\liminf _{h \rightarrow 0^{+}} \delta_{t, t-h}[f](x)=\underline{A}_{t}[f](x) .
$$

1) For Lemma 3.2, we have first to obtain the equivalent of (24). Using [Continuity], we construct a function $\tilde{\phi}$ as in Lemma 3.2 and write

$$
T_{t_{0}-h, 0}\left[u_{0}\right] \leqslant \tilde{\phi}\left(t_{0}-h, \cdot\right) \quad \text { for } h<t_{0} .
$$

Applying $T_{t_{0}, t_{0}-h}$, [Monotonicity] and [Causality], we get

$$
T_{t_{0}, 0}\left[u_{0}\right]-u\left(t_{0}, x_{0}\right) \leqslant T_{t_{0}, t_{0}-h}\left[\tilde{\phi}\left(t_{0}-h, \cdot\right)\right]-\tilde{\phi}\left(t_{0}, x_{0}\right) .
$$

By the same computation, using [Regularity], we obtain

$$
\begin{aligned}
0 \leqslant & T_{t_{0}, t_{0}-h}\left[\tilde{\phi}\left(t_{0}, \cdot\right)\right]\left(x_{0}\right)-\tilde{\phi}\left(t_{0}, x_{0}\right)-h\left[\frac{\partial \tilde{\phi}}{\partial t}\left(t_{0}, x_{0}\right)+\varepsilon\left(h, x_{0}\right)\right] \\
& +m\left(t_{0}, x_{0}, \tilde{\phi}\left(t_{0}, \cdot\right), \frac{\partial \tilde{\phi}}{\partial t}\left(t_{0}, \cdot\right)+\varepsilon(h, \cdot), h\right) h .
\end{aligned}
$$

This inequality provides the equivalent one of Lemma 3.2.

2) The proof of Lemma 3.1 is the same and in proof of Lemma 3.3, we just have to use [Stability] in order to obtain the time-continuity for $\underline{F}$ and $\bar{F}$ deduced from $\underline{A}$ and $\bar{A}$.

3) For Lemma 3.4 we observe in addition that the same arguments show that if $t_{0}$ is fixed and $u_{0} \in X,(h, x) \mapsto T_{t_{0}+h, t_{0}}\left[u_{0}\right](x)$ is a continuous vicosity solution of

$$
\frac{\partial u}{\partial h}+\bar{F}\left(t_{0}+h, x, u(h, x), D u(h, x), D^{2} u(h, x)\right)=0
$$

and also of the same equation with $\bar{F}$ replaced by $\underline{F}$. 
4) In the proof of the Lemma 3.5, we fix $\left(t_{0}, x_{0}, r, p, M\right) \in[0,+\infty) \times \mathbb{R}^{N} \times \mathbb{R} \times \mathbb{R}^{N} \times$ $\mathcal{S}(N)$ and use the function

$$
(h, x) \mapsto T_{t_{0}+h, t_{0}}[f](x),
$$

where $f=f_{x_{0}, r, p, M}$ which gives a viscosity solution of (22). Using this fact, we obtain that for $h>0$ small enough,

$$
T_{t_{0}+h, t_{0}}[f]\left(x_{0}\right) \leqslant f\left(x_{0}\right)+h\left[-\underline{F}\left(t_{0}, x_{0}, r, p, M\right)+\delta\right] .
$$

We then use [Stability] with $t=t_{0}$ and $t^{\prime}=t_{0}-h$ to get

$$
T_{t_{0}, t_{0}-h}[f]\left(x_{0}\right)-f\left(x_{0}\right)-h \cdot p_{x_{0}, f}(h) \leqslant h\left[-\underline{F}\left(t_{0}, x_{0}, r, p, M\right]+\delta\right]
$$

and we obtain the result dividing by $h$, taking the lim sup and letting $\delta$ go to 0 .

\section{Appendix A}

We give here the proof of Lemma 3.2 we have postponed in Section 3.

Proof of Lemma 3.2. - We make the proof for $(a, p, M) \in \mathcal{P}^{2,+}[u]\left(t_{0}, x_{0}\right)$, the one for $(a, p, M) \in \mathcal{P}^{2,-}[u]\left(t_{0}, x_{0}\right)$ being the same with straightforward adaptations.

1) We first construct a suitable function. Since $(a, p, M) \in \mathcal{P}^{2,+}[u]\left(t_{0}, x_{0}\right)$, by classical results, there exists $\phi \in C^{\infty}\left((0,+\infty) \times \mathbb{R}^{N}\right)$ such that $u-\phi$ has a local maximum point at $\left(t_{0}, x_{0}\right)$ with $\phi\left(t_{0}, x_{0}\right)=u\left(t_{0}, x_{0}\right), D \phi\left(t_{0}, x_{0}\right)=p, D^{2} \phi\left(t_{0}, x_{0}\right)=M$ and $\frac{\partial \phi}{\partial t}\left(t_{0}, x_{0}\right)=a$ (see for example [3]). There exist $\alpha, r>0$ such that

$$
u \leqslant \phi \quad \text { on }\left[t_{0}-\alpha, t_{0}+\alpha\right] \times \overline{B\left(x_{0}, r\right)} .
$$

Recalling that $u(t, x)=T_{t}\left[u_{0}\right](x)$, [Continuity] provides a function $f_{u_{0}, t_{0}, \alpha}$ such that

$$
u(t, x) \leqslant f_{u_{0}, t_{0}, \alpha}(x) \quad \text { for } t \in\left[t_{0}-\alpha, t_{0}+\alpha\right], x \in \mathbb{R}^{N} .
$$

Taking $v$ as in proof of Lemma 3.5, we construct $v_{r}(x):=v\left(\left(x-x_{0}\right) / r\right)$, and get

$$
u \leqslant \phi v_{r}+\left(1-v_{r}\right) f_{u_{0}, t_{0}, \alpha}=\tilde{\phi} \quad \text { on }\left[t_{0}-\alpha, t_{0}+\alpha\right] \times \mathbb{R}^{N} .
$$

Since $X$ is assumed to be a vector space containing $\mathcal{D}\left(\mathbb{R}^{N}\right), \tilde{\phi}(t, \cdot) \in C_{X}^{\infty}\left(\mathbb{R}^{N}\right)$ for $t \in\left[t_{0}-\alpha, t_{0}+\alpha\right]$. Moreover, $\tilde{\phi} \equiv \phi$ on $\left[t_{0}-\alpha, t_{0}+\alpha\right] \times B\left(x_{0}, r / 2\right)$. Thus

$$
\left(\tilde{\phi}\left(t_{0}, x_{0}\right), \frac{\partial \tilde{\phi}}{\partial t}\left(t_{0}, x_{0}\right), D \tilde{\phi}\left(t_{0}, x_{0}\right), D^{2} \tilde{\phi}\left(t_{0}, x_{0}\right)\right)=\left(u\left(t_{0}, x_{0}\right), a, p, M\right) .
$$

2) We rewrite (23), for $h$ sufficiently small, under the form

$$
T_{t_{0}-h}\left[u_{0}\right] \leqslant \tilde{\phi}\left(t_{0}-h, \cdot\right) .
$$


Then using [Monotonicity] and substracting $u\left(t_{0}, x_{0}\right)=\tilde{\phi}\left(t_{0}, x_{0}\right)$ we obtain, for $h>0$ sufficiently small

$$
T_{h}\left[T_{t_{0}-h}\left(u_{0}\right)\right]-u\left(t_{0}, x_{0}\right) \leqslant T_{h}\left[\tilde{\phi}\left(t_{0}-h, \cdot\right)\right]-\tilde{\phi}\left(t_{0}, x_{0}\right)
$$

Using now [Causality] and applying the inequality at $x_{0}$, this yields

$$
0 \leqslant T_{h}\left[\tilde{\phi}\left(t_{0}-h, \cdot\right)\right]\left(x_{0}\right)-\tilde{\phi}\left(t_{0}, x_{0}\right) .
$$

Then by Taylor's formula for $\tilde{\phi}\left(t_{0}-\cdot, x\right)$

$$
0 \leqslant T_{h}\left[\tilde{\phi}\left(t_{0}, \cdot\right)-h \frac{\partial \tilde{\phi}}{\partial t}\left(t_{0}, \cdot\right)-h \varepsilon(h, \cdot)\right]\left(x_{0}\right)-\tilde{\phi}\left(t_{0}, x_{0}\right)
$$

We notice that, by construction of $\tilde{\phi}, w(h, \cdot)=\frac{\partial \tilde{\phi}}{\partial t}\left(t_{0}, \cdot\right)+\varepsilon(h, \cdot) \in \mathcal{D}\left(\mathbb{R}^{N}\right)$ and apply [Regularity] in the last inequality. We get

$$
0 \leqslant T_{h}\left[\tilde{\phi}\left(t_{0}, \cdot\right)\right]\left(x_{0}\right)-\tilde{\phi}\left(t_{0}, x_{0}\right)-h\left[\frac{\partial \tilde{\phi}}{\partial t}\left(t_{0}, x_{0}\right)+\varepsilon\left(h, x_{0}\right)\right]+m_{x_{0}, \tilde{\phi}\left(t_{0}, \cdot\right), w(h, \cdot)}(h) h .
$$

Remarking that the functions $(w(h, \cdot))_{t_{0} \geqslant h \geqslant 0}$ are in a same $Q_{d}$ independent of $h$ for some sequence $d$, we deduce from (24) that

$$
\limsup _{h \rightarrow 0^{+}} \delta_{h}\left[\tilde{\phi}\left(t_{0}, \cdot\right)\right]\left(x_{0}\right) \geqslant \liminf _{h \rightarrow 0^{+}} \delta_{h}\left[\tilde{\phi}\left(t_{0}, \cdot\right)\right]\left(x_{0}\right) \geqslant \frac{\partial \tilde{\phi}}{\partial t}\left(t_{0}, x_{0}\right) .
$$

And the proof is complete.

\section{REFERENCES}

[1] Alvarez L., Guichard F., Lions P.L., Morel J.M., Axioms and Fundamental Equations of Image Processing, Arch. Rational Mech. Anal. 123 (1993) 199-257.

[2] Bardi M., Capuzzo-Dolcetta I., Optimal Control and Viscosity Solutions of HamiltonJacobi-Bellman Equations, Birkhäuser, Boston, 1997.

[3] Barles G., Solutions de Viscosité des Équations de Hamilton-Jacobi, Collection "Mathématiques et Applications" of SMAI, No 17, Springer-Verlag, Paris, 1994.

[4] Beck A., Uniqueness of Flow Solutions of Differential Equations, Lecture Notes in Mathematics, Vol. 318, Springer-Verlag, Berlin, 1973.

[5] Crandall M.G., Lions P.-L., Viscosity solutions of Hamilton-Jacobi equations, Trans. Amer. Math. Soc. 277 (1983) 1-42.

[6] Crandall M.G., Ishii H., Lions P.-L., User's guide to viscosity solutions of second order Partial differential equations, Bull. Amer. Soc. 27 (1992) 1-67.

[7] Fleming W.H., Soner H.M., Controlled Markov Processes and Viscosity Solutions, Applications of Mathematics, Springer-Verlag, New York, 1993.

[8] Giga Y., Goto S., Ishii H., Sato M.-H., Comparison principle and convexity preserving properties for singular parabolic equations on unbounded domains, Indiana University Math. J. 40 (2) (1991). 
[9] Ishii I., Uniqueness of unbounded viscosity solution of Hamilton-Jacobi equations, Indiana Univ. Math. J. 33 (1984) 721-748.

[10] Ley O., Lower bound gradient estimates for first-order Hamilton-Jacobi equations and applications to the regularity of propagating fronts, Preprint, 1999.

[11] Lions P.-L., Generalized Solutions of Hamilton-Jacobi Equations, Research Notes in Mathematics, Vol. 69, Pitman, London, 1988.

[12] Lions P.-L., Some Properties of the Viscosity Semigroups of Hamilton-Jacobi Equations, Nonlinear Differential Equations and Applications, Pitman, London, 1982. 